\title{
The Ingredients of Effective Social Skills Programming for Children and Adolescents on the Autism Spectrum: A Synthesis of Meta-Analytical Research
}

\author{
Scott Bellini' ${ }^{*}$ and Olivia Heck²
}

*Correspondence: sbellini@indiana.edu

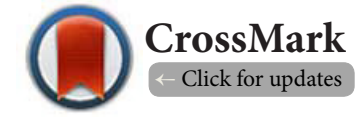

${ }^{1}$ Associate Professor, School Psychology, Indiana University, Bloomington, 201 N. Rose Ave. Bloomington, IN 47405, USA.

${ }^{2}$ Graduate Student, School Psychology, Indiana University, Bloomington, 201 N. Rose Ave. Bloomington, IN 47405, USA.

\begin{abstract}
Difficulties with social skills and developing social relationships is a key diagnostic characteristic of autism spectrum disorders, and is often an enduring and pervasive issue throughout the life of a person on the autism spectrum. Previous research has demonstrated a clear link between social skill deficits and numerous negative developmental outcomes. The development of effective social interaction skills is critical to successful social, emotional, language, and cognitive development, yet research on social skills programming has produced conflicting results related to the effectiveness of many social skill interventions. The purpose of this article is to synthesize the results of both literature reviews and metaanalytical studies on social skills programing for children and adolescents on the autism spectrum. The primary purpose of the article is to elucidate the ingredients of effective social skills programming for children on the autism spectrum. This article will review the existing meta-analytical research relevant to social skills programming and provide recommended practices for youth on the autism spectrum. The article will also provide suggestions for future researchers to consider when designing studies on social skills programming. The present article will provide useful guidance to both practitioners and researchers.
\end{abstract}

Keywords: Autism, social skills, intervention

\section{Background}

Social skill impairment is a pervasive and enduring feature of autism spectrum disorders that significantly impacts numerous developmental outcomes. Social interaction skills are essential to successful social, emotional, language, and cognitive development. Poor social skills have been linked to numerous negative life outcomes, such as social failure, peer rejection, bullying (for both the bully and victim), anxiety, depression, substance abuse, suicidal ideation, delinquency, and other forms of psychopathology [1-3]. The results of longitudinal research indicate that social skills entering kindergarten are strong predictors of employment, criminal behavior, mental health, substance abuse, and educational attainment in young adulthood [4]. In addition, positive social skills have been associated with positive academic outcomes, such as improved grades and test scores [5]. Effective social skills provide an opportunity for children to engage in positive interactions with peers and adults, as well as establish meaningful social relationships. These social relationships provide opportunities for continued community integration and serve as sources of social support during difficult times in the person's life [6]. Clearly, effective social skill interventions have the potential to positively impact the lives of children and adolescents on the autism spectrum, and improve developmental outcomes. However, effective social skills programming is often an elusive component of many children's educational plans.

A number of literature reviews and meta-analytical studies have been conducted to better understand the outcomes of social skill interventions for children and adolescents. These literature reviews and meta-analyses have produced wide variability in the reported outcomes of social skill interventions. Authors of narrative reviews generally conclude that social skills programming is an effective treatment modality. In contrast, the majority of meta-analytical studies have reported mild 
Bellini et al., Journal of Autism 2019,

http://www.hoajonline.com/journals/pdf/2054-992X-6-3.pdf

doi: 10.7243/2054-992X-6-3

to poor treatment, maintenance, and generalization effects for social skill interventions. The purpose of this article is to synthesize the results of these literature reviews and metaanalytical studies to elucidate the ingredients of effective social skills programming for children on the autism spectrum. This article will provide a review of the existing meta-analytical research relevant to social skills programming and provide recommended intervention and research practices for youth on the autism spectrum.

\section{Review}

\section{Ingredients of Effective Social Skills Programming}

Numerous qualitative reviews have examined the effectiveness of social skill interventions for children and adolescents with ASD, and have provided suggestions for increasing the effectiveness of social skills programming [7-13]. A limitation of these narrative reviews is a lack of a quantitative metric to evaluate treatment effectiveness. That is, the researchers often report conclusions drawn by the studies' authors in regards to treatment effectiveness without conducting a systematic analysis themselves, such is the case in meta-analytical reviews. Meta-analytical studies also allow researchers to elucidate setting, participant, and procedural features that lead to the most beneficial outcomes for participants. These metaanalytical reviews provide relative comparisons of treatment effectiveness across different intervention strategies. A number of quantitative meta-analyses have been performed on social skill intervention studies involving children and adolescents with and without ASD [14-22]. In general, these studies have produced conflicting results on the effectiveness of social skill interventions for children with disabilities. However, taken together, the collective outcomes of these studies provide helpful guidance in determining the ingredients of effective social skills programming for children on the autism spectrum.

Though there is wide variability in the reported outcomes of social skill training in various literature reviews and metaanalytical studies, a review and synthesis of these articles allows us to elucidate the ingredients to effective social skills programming. The following recommendations for effective social skills training have emerged from this research synthesis, and are discussed in this article:

1. Increase the dosage of social skill interventions

2. Provide instruction within the child's natural environment

3. Conduct a comprehensive social skills assessment

4. Develop clear and measureable treatment objectives

5. Match the intervention strategy with the type of skill deficit

6. Facilitate the generalization of skills across settings and persons

7. Ensure intervention fidelity and social validity

8. Implement systematic social skills programming

\section{Increase the dosage of social skill interventions}

The length and duration of the intervention is a critical component of effective social skills programming. Gresham, Sugai, and
Horner [16] concluded that many social skills programs fail because the intensity and frequency of instruction is simply insufficient to meet the substantial needs of the target child. Though the researchers did not recommend a specific dosage, they stated that interventions should exceed 30 hours of instruction over a 10-12 week period. The low intervention effects observed in the previous meta-analyses may be attributed to the low level of instructional intensity provided in the reviewed studies, which were all considerably lower than the 30+ hours recommended over a 10-12 week period. Bellini, Peters, Benner and Hopf [14] conducted a meta-analysis of 55 school based social intervention studies involving students on the autism spectrum. The median hours of intervention in these studies was only 7.25 hours, and none reached the dosage recommendation of Gresham, Sugai, and Horner [16]. It is important to note that most of the reviewed meta-analytical studies found no significant relationships between length of intervention and intervention outcomes. However, most studies either documented a very low treatment dosage and intensity, or simply failed to provide adequate descriptive information regarding the intervention length and duration $[14,15,17]$. That is, we cannot determine the true effects of treatment dosage because most studies fail to either meet the recommended dosage or fail to report hours of intervention in the published manuscript. Therefore, results related to intervention length and study outcomes should be interpreted with caution.

As previously noted, children on the autism spectrum exhibit substantial and pervasive social skill deficits and often fail to learn social skills implicitly during naturalistic exposure to social situations and other children [23]. Many children on the autism spectrum experience a lifetime of social difficulties. It stands to reason that a mere 30-45 minutes of social skills programming per week will not be of a sufficient intensity to change their developmental course and produce meaningful positive outcomes. As such, the recommendation to increase instructional intensity is particularly salient for this population of children. School personnel and teachers should look for opportunities to teach and reinforce social skills throughout the day and across multiple settings to ensure adequate dosage and intensity of their social skills programming.

Provide Instruction in the Child's Natural Environment The location in which social skills are taught has a substantial impact on the outcomes of the program. According to Gresham, Sugai, and Horner, the weak outcomes of social skills programming can be attributed to the fact that these interventions often take place in "contrived, restricted, and decontextualized" (p. 340) settings, such as resource rooms or other "pull-out" settings [16]. In other words, we often remove children from rich social environments to teach them social skills in contrived settings. This "decontextualized" programming leads to poor maintenance and generalization effects. The results of the Bellini, Peters, Brenner, and Hopf [14] meta-analysis support this assertion. Maintenance and generalization effects were 
significantly and substantially lower for interventions that were implemented in "pull-out" settings only. In contrast, interventions that were implemented in the child's typical classroom setting, at least part of the time, produced higher maintenance effects and higher generalization effects across persons, settings, and play stimuli. Furthermore, in addition to higher maintenance and generalization effects, results of the meta-analysis suggest that interventions implemented in the child's typical classroom produce higher direct intervention effects [14].

This finding has important implications for school-based social skills programming. Social skills programming should not stop the moment the child leaves the resource room or therapist's office. As such, teachers and other school personnel should place a premium on selecting social skill interventions that can be reasonably implemented within multiple naturalistic settings. This is particularly important for children on the autism spectrum who may have considerable difficulties transferring skills from one setting to another. This recommendation is not meant to dissuade school personnel and therapists from teaching social skills in "pull-out" or other contrived settings. The lesson gleaned from meta-analytical research is that social skill interventions should not only be taught in "pull-out" settings. Indeed, school personnel, therapists, and parents should look for opportunities to teach social skills in every environment that the child enters [23].

\section{Conduct a Comprehensive Social Skill Assessment}

Evaluation of social skills and social competence is a critical element of social skills training, yet many social skills programs fail to adequately assess social skills prior to implementing the intervention $[\mathbf{1 4}, \mathbf{1 6}]$. There are two primary goals of the social skills assessment. One goal is to identify specific social skill deficits that will be the direct target of the intervention. The second is to evaluate the outcomes of the social skills program. Research studies on social skill interventions often include the latter (i.e., an objective measures of change), but routinely omit the former (i.e., a pre-assessment to identify skills to target). None of the 55 studies reviewed in the Bellini et al. meta-analysis conducted an assessment of social skills prior to determining the skills to be taught in the intervention. Instead, these studies selected target behaviors a priori, without regard to the unique skill profiles and social skill deficits of the children participating in the studies. In a meta-analysis of 53 studies examining social skills training for youth with learning disabilities, Forness and Kavale [15] concluded that the low treatment effects associated with social skill interventions can be attributed to the use of poorly conceptualized assessment tools that fail to link the social skills being assessed with the social skills being taught.

Gresham [24] divides social skills assessment methods into three categories that measure different aspects of social functioning. Type I measures include rating scales and interviews designed to measure social competence. Treatment objectives developed from Type I measures are likely to be accepted and viewed as socially acceptable by key stakeholders. A major advantage of Type I measures is their ability to quickly and efficiently obtain information regarding social behavior from a variety of sources and across a variety of settings. A major disadvantage of Type I measures is the fact that they are often not sensitive to short term changes in behavior. For instance, the child might be demonstrating an increase in the target behaviors without key stakeholders noticing these changes. Type II measures involve the direct observation of the child's social skills. As such, these measures are valuable for progress monitoring and are used extensively in applied research studies involving single case design research. Type II measures are sensitive to small changes in behavior because they are linked directly to the treatment objectives. For instance, if the interventionist identifies "responding to the initiations of peers" as a treatment objective, the interventionist would then observe the child directly to measure whether "responding" behavior has increased over the course of the intervention. Type III measures involve conducting role-play scenarios or asking questions related to social cognitive processing (e.g., social problem solving, self awareness, or perspective taking scenarios). Type III measures are the least reliable and valid assessment measures and are often difficult to administer [24]. Though these are important areas to address via intervention, research has demonstrated that these measures are not related to measures of social competence (Type I measures) or measures of social skills (Type II measures).

Develop Clear and Measurable Intervention Objectives The use of reliable and valid assessment procedures leads to the development of clear and measureable intervention objectives, and the identification of social skills that will be the target of the subsequent intervention [23]. Gresham and colleagues [16] concluded that the traditionally weak treatment effects of many social skills programs might be the result of interventions that fail to link assessment data with the development of intervention objectives. Objectives refer to the outcome variables of the social skills program. That is, they determine how the interventionist will define and measure progress in the social skills program. An advantage of clear and measurable intervention objectives is that they also allow the interventionist to clearly identify the precise skills that will be targeted in the intervention. Quinn and colleagues [18] found that social skills programs that targeted specific social skills (e.g., turn taking, social initiations) were more effective than programs that focused on more global social functioning, such as "friendship" skills, cooperation, sharing, or other non-specific behaviors. Likewise, Bellinger, Perlman, and DiPerna [8] concluded that social skills programs should clearly identify the objectives and targeted skills of the intervention prior to selecting an intervention strategy. This gap between assessment practices and intervention objectives leads to vague and ambiguous treatment objec- 
Bellini et al., Journal of Autism 2019,

http://www.hoajonline.com/journals/pdf/2054-992X-6-3.pdf

doi: 10.7243/2054-992X-6-3

tives and may significantly diminish both the social validity and effectiveness of the intervention.

Objectives are connected to the data collected during the Type I and Type II assessment, and allow the interventionist to determine the skills that should be targeted during the intervention. For instance, if the interventionist selects "increasing positive social engagement with peers" as an objective of the intervention, the next step would be to determine the precise social skills and social cognitive processes that the child will need to learn and perform in order to be successful on the objective. In this case, the assessment data might indicate that positive engagement is impacted by the fact that the child is failing to join activities with peers, or failing to self-monitor his behavior during interactions. As such, the intervention would then directly target these skill deficits (and others as determined by the assessment) so that the child will successfully reach the objective of "increasing positive engagement with peers." This logical link between assessment, intervention objectives, and targeted skills is critical for successful social skills programming.

\section{Match the Intervention with the Type of Skill Deficit}

A key component of effective social skills programming is the ability of the interventionist to match the intervention strategy with the type of skill deficit exhibited by the child [16]. In social skills programming, there are two primary types of skill deficits for which the interventionist should be cognizant: skill acquisition deficit and performance deficit. A skill acquisition deficit refers to the absence of a particular skill or behavior. A child with a skill acquisition deficit fails to perform a skill or behavior because she does not have the skill in her current repertoire. A performance deficit on the other hand, refers to a skill or behavior that is in the child's repertoire, but not demonstrated or performed. Quinn, Kavale, Mathur, Rutherford Jr., and Forness [18] concluded that the failure of many social skill interventions is a result of a mismatch between type of strategy and type of skill deficit. Of the 55 studies included in the Bellini et al. [14] meta-analysis, only one identified the type of skill deficit exhibited by the participants prior to selecting intervention strategies.

Identifying the type of skill deficit guides the selection of intervention strategies [23]. As such, school personnel and therapists should make a concerted effort to systematically match the intervention strategy to the type of skill deficits exhibited by the child. For instance, if the child lacks the skills necessary to joinin an interaction with peers (skill acquisition deficit), a strategy should be selected that promotes skill acquisition, or teaches the new skill. In contrast, if the child has the skills to join-in an activity but regularly fails to do so (performance deficit), a strategy should be selected that enhances performance of the existing skill. It would be ineffective and unnecessary to subject the child to intensive social skills programming designed to teach new skills, when, in fact the child already has the skills in her repertoire. Like- wise, performance enhancement strategies will be infective if the child does not already have the skills in her repertoire to be successful. For instance, if the child does not have the necessary skills to effectively maintain an interaction with peers, simply providing positive reinforcement contingent upon the child interacting more frequently would be inadequate and ineffectual. Instead, the child would need to be systematically taught the skills necessary to successfully maintain the interaction with peers.

\section{Facilitate the Generalization of Skills across Settings and Persons}

The ultimate goal of social skills programming should be to teach the child to interact successfully with multiple persons and across multiple naturalistic settings. Gresham and colleagues [16] concluded that a persistent weakness in social skills training programs is their failure to demonstrate adequate generalization effects. This is primarily a result of interventions that fail to plan for generalization, which refers to the transfer of skills across settings, persons, situations, and time. In the Bellini [14] meta-analysis, only 15 of the 55 reviewed studies measured generalization effects. And those that did measure generalization produced very low generalization effects. An essential goal of all social skills programs should be to develop and implement a plan for generalization.

In social skills programming, generalization should be considered from both behavioral and cognitive perspectives. From a behavioral perspective, the inability to generalize a skill or behavior is a result of training that is too inflexible in its use of discriminative stimuli. That is, the child learns to only perform a particular skill or behavior in the presence of a specific stimulus (person, prompt, directives, etc.). Generalization is particularly important for children with ASD who often have pronounced difficulties transferring skills across persons and settings. A number of strategies may be used to facilitate generalization of social skills across settings, persons, situations, and time, including [23]: (a) Reinforce the performance of social skills in the natural environment, (b) Train with multiple persons and in multiple settings, (c) Ensure the presence and delivery of natural reinforcers for the performance of social skills, (d) Practice the skill in the natural environment, (e) Fade prompts as quickly as feasible, (f) Provide multiple exemplars for social rules and concepts, (g) Train skills "loosely" (i.e., vary the instruction, directives, strategies, and prompts used during skill instruction), (h) Teach self-monitoring strategies, and (i) provide "booster" sessions (i.e., provide follow-up training after initial instruction has been discontinued).

Failure to generalize skills can also be explained from a cognitive perspective. According to cognitive theorists [25], generalization of skills fails to occur because the child does not recognize that a skill she has learned can be used in a new situation, or with different people. Or, she may recognize that the skill can be used, but she has difficulty using it because 
she lacks mastery or fluency with the skill. Another reason why children fail to generalize skills is that they do not think that the outcome is worth the effort required to obtain it. That is, if the child has no interest or motivation in interacting with peers, she probably will not exert much effort to be part of a social interaction. Finally, failure to generalize skills also occurs when the child does not understand why the strategy is useful and how the strategy might be beneficial to them. Based on this cognitive perspective on failure to generalize skills, the following suggestions are provided to promote generalization of social skills [23]: (a) Encourage application of skills in multiple situations, (b) Increase mastery of skill performance, (c) Encourage and facilitate an interest in interacting with others and developing relationships, (d) Build self-efficacy related to social performance, and (e) Provide information on why the skill is useful.

\section{Ensure Intervention Fidelity and Social Validity}

Intervention fidelity refers to methods to ensure that intervention strategies are implemented as intended. Only 14 of the studies in the Bellini [14] meta-analysis measured whether the intervention was implemented as intended. Gresham, Sugai, and Horner [16] concluded that the failure of studies to provide intervention fidelity data makes it extremely difficult to conclude whether a social skill intervention was ineffective because of an ineffectual intervention strategy, or because the strategy was implemented poorly. Poor intervention fidelity may significantly diminish the outcomes of the social skill intervention, and diminish our ability to make decisions regarding the effects of individual strategies. One area that could impact intervention fidelity is the fact that few educators have had formal training on social-emotional instruction as part of their pre-service education programs [26]. As demands to teach social-emotional functioning increase, it is imperative that educators and therapists are provided numerous professional development opportunities to address this gap in pre-service training.

Social validity is also an important aspect to consider in social skills programming. Social validity refers to the social significance of the treatment objectives, the social significance of the intervention strategies, and the social importance of the intervention results [27]. Thus, it involves ensuring that the consumers (parents, teachers, and child) believe that the selected intervention strategies are effective and appropriate, and that the social objectives are important for the child to achieve. Social validity data were only collected in 12 of the 55 studies in the Bellini meta-analysis [14]. Allowing consumers to continually provide input and feedback on the goals and strategies of the intervention is essential to enhancing social validity. Social validity also has a direct impact on intervention fidelity. If the intervention lacks social validity, consumers are less likely to exert the effort necessary to implement the intervention, thus diminishing intervention fidelity. School personnel and researchers should ensure that their interven- tions are socially valid, and make a concerted effort to collect data related to both intervention fidelity and social validity throughout their interventions.

\section{Implement Systematic Social Skills Programming}

Viewed collectively, the seven ingredients discussed above highlight the importance of developing and implementing social skills programming more systematically. Social skills programming is most effective when it is implemented as part of a methodical plan, with sufficient thoroughness and regularity, and when skills are explicitly taught to the child $[12,18,23]$. White, Keonig, and Scahill [12] conducted a literature review of 14 group social skills intervention studies for youth on the autism spectrum. The authors concluded that group social skills training could be an effective modality for improving social behaviors, but only when skills are explicitly taught to the child.

Similarly, Quinn and colleagues [18] concluded that interventions should be designed to systematically address the individual needs of the child, rather than forcing the child to "fit" into an intervention selected a priori, with little regard for the child's particular needs. Unfortunately, systematic social skills programming is not the norm, especially in school settings [23]. Schools often have no organized plan for teaching social skills. Although social objectives are commonly developed for students on the autism spectrum, they are rarely based on a reliable and valid assessment of social functioning that takes into considerations the child's unique skills profile. Instead, they are based on a generic diagnostic description, and interventions take on a "one size fits all" model. Furthermore, seldom do social skill interventions proceed in a methodical or systematic fashion. Commonly, social skills programming is relegated to inferior status and only implemented when teachers and other school practitioners have the extra time to address it.

Bellini and colleagues $[\mathbf{2 3}, \mathbf{2 8}, \mathbf{2 9}]$ provide an example of a systematic and research-based approach for teaching social interaction skills to youth on the autism spectrum that incorporates the ingredients outlined previously in this manuscript. The program follows the following five-step plan:

1. Assess Social Functioning

2. Distinguish Between Skill Acquisition and Performance Deficits

3. Select Intervention Strategies

4. Implement Intervention

5. Evaluate and Monitor Progress

The first step of the program consists of conducting a thorough assessment of the individual's current level of social skills functioning that also identifies the social skills that will be targeted during the intervention. A social skills assessment includes Type I, Type II, and Type III measures and is administered at the beginning, middle, and at the conclusion of the program. The results of the initial assessment are used to develop clear and measurable intervention objectives. After 
Bellini et al., Journal of Autism 2019,

http://www.hoajonline.com/journals/pdf/2054-992X-6-3.pdf

doi: 10.7243/2054-992X-6-3

the assessment is complete, and objectives are developed, the next step is to discern between skill acquisition deficits and performance deficits. This information allows the interventionist to focus the program on either skill acquisition or performance enhancement. Based on this information, the third step of the program, selection, and implementation of intervention strategies, takes place.

The strategies of the program are divided into two categories: strategies that promote the acquisition of new skills (video self-modeling, direct instruction, social narratives, social problem solving, etc.) and strategies that enhance or increase the performance of existing skills (positive reinforcement, prompting, priming, peer-mediated instruction, etc.). It is imperative to program success that the strategies are matched to the unique needs of the student, and to the nature of the skill deficits. The program contains 13 of the evidence-based social skill strategies identified by both the National Professional Development Center for Autism and the National Standards Report published by the National Autism Center [30-31]: video-modeling, modeling, social narratives, naturalistic interventions, visual supports, peer mediated instruction, parent implemented interventions, self-management, prompting, time-delay prompt fading, structured play groups, cognitive behavioral intervention, positive reinforcement, and social skills groups.

The fourth step of the program involves the implementation phase of the program. This step involves the planning and implementation stages that include structuring and developing lesson plans, and determining where the sessions will take place, and with whom the child will participate. The program may be implemented in individual, small group, and class-wide formats, and takes place in multiple settings (including both pull-out and naturalistic). All small group sessions include the use of peer mentors and involve parent and/ or teacher training to ensure that social skills are taught and reinforced in the natural environment to ensure that instruction is of a sufficient intensity. The final step of the program is to evaluate progress using both summative and formative assessment procedures. The program is divided into 9-week sessions, which allows for frequent progress monitoring and plan modifications. Intervention objectives are measured at the beginning and end of each 9-week period in both the treatment (therapy room, resource room, classroom, etc.) and generalization setting (playground, home, community, etc.). In addition to summative assessment data, formative data are collected on both social validity and treatment fidelity throughout the intervention.

\section{Implications for Future Research}

In addition to the practical implications for educators and practitioners discussed above, these recommendations also provide useful guidance to researchers. First, researchers should provide detailed descriptions of intervention dosage, and future research should explicitly examine the relative ef- fectiveness of interventions that exceed the recommended dosage of 30 hours over a 10-12 week period. Future research should also be conducted to further examine the differences between interventions conducted in contrived settings versus interventions conducted in naturalistic settings. For instance, comparison studies can be conducted to examine the differential effects of identical interventions implemented across multiple settings.

Additional research is also needed to examine the outcomes of interventions that systematically match strategies to the type of skill deficits. In addition, it would be useful to practitioners if researchers could precisely identify and categorize strategies based on the skill acquisition and performance deficit dichotomy. This would allow practitioners to efficiently select strategies that match the child's particular skill deficit. Finally, the field of social skills programing is in critical need of more reliable and valid Type III assessment measures. As such, researchers should seek to develop more psychometrically sound Type III measures related to various facets of social cognitive processing, such as social problem solving, perspective, self-awareness, and attentional processing.

\section{Summary}

The purpose of this article was to elucidate the ingredients of effective social skills programming for youth on the autism spectrum. The article provided a synthesis of the findings of literature reviews and meta-analytical studies that have previously been conducted on social skill interventions for children and adolescents. Social skills programming must begin with a comprehensive assessment that leads to the formulation of clear and measurable treatment objectives. Social skills programming should include social skill assessment measures with adequate precision and established psychometric properties. It is also imperative to identify the precise skills that will be taught during the intervention and also determine if the type of skill deficits are exhibited by the target child. Results of previous meta-analytical research indicate that social skills programming for youth on the autism spectrum must be intensive, and should be implemented (at least partly) in the child's natural environment. However, intensity and location of instruction are only important if the intervention itself is effective. Further, social skills programming should explicitly plan for the generalization of skills across settings and persons. The present article also highlights the importance of teaching social skills in a systematic and purposeful manner. Finally, future research should be designed to examine the relative benefits of each of these ingredients of effective social skills programming, and further evaluate the participant, procedural, and setting variables related to the most beneficial outcomes for children on the autism spectrum.

\section{Competing interests}

The authors declare that they have no competing interests. 
Bellini et al., Journal of Autism 2019,

http://www.hoajonline.com/journals/pdf/2054-992X-6-3.pdf

Authors' contributions

\begin{tabular}{|l|c|c|}
\hline Authors' contributions & SB & OH \\
\hline Research concept and design & $\checkmark$ & -- \\
\hline Collection and/or assembly of data & $\checkmark$ & -- \\
\hline Data analysis and interpretation & $\checkmark$ & -- \\
\hline Writing the article & $\checkmark$ & -- \\
\hline Critical revision of the article & $\checkmark$ & $\checkmark$ \\
\hline Final approval of article & $\checkmark$ & $\checkmark$ \\
\hline
\end{tabular}

Publication history

Editor: David Reiss, Imperial College London, UK.

Received: 24-Oct-2019 Final Revised: 25-Nov-2019

Accepted: 28-Nov-2019 Published: 14-Dec-2019

\section{References}

1. Bellini S. The development of social anxiety in high functioning adolescents with autism spectrum disorders. Focus Autism Other Dev Disabl. 2006; 21:138-145. | Article

2. Cook CR, Williams KR, Guerra NG, Kim TE and Sadek S. Predictors of bullying and victimization in childhood and adolescence: a meta-analytic investigation. School Psychology Quarterly. 2010; 25:65-83. | Article

3. Tantam D. Psychological disorder in adolescents and adults with Asperger syndrome. Autism. 2000; 4:47-62. | Article

4. Jones DE, Greenberg M and Crowley M. Early Social-Emotional Functioning and Public Health: The Relationship Between Kindergarten Social Competence and Future Wellness. Am J Public Health. 2015; 105:2283-90. | Article | PubMed Abstract | PubMed FullText

5. Durlak JA, Weissberg RP, Dymnicki AB, Taylor RD and Schellinger KB. The impact of enhancing students' social and emotional learning: a meta-analysis of school-based universal interventions. Child Dev. 2011; 82:405-32. | Article | PubMed

6. Cohen S. Social relationships and health. Am Psychol. 2004; 59:676-684. | Article | PubMed

7. Hwang $B$ and Hughes $C$. The effects of social interactive training on early social communicative skills of children with autism. J Autism Dev Disord. 2000; 30:331-43. | Article | PubMed

8. Bellinger, JM, PerIman, EH and DiPerna JC. Social skill interventions for individuals with autism spectrum disorders. School Psychology Forum. 2011; 5:141-159.

9. McConnell SR. Interventions to facilitate social interaction for young children with autism: review of available research and recommendations for educational intervention and future research. $J$ Autism Dev Disord. 2002; 32:351-72. | Article | PubMed

10. Krasny L, Williams BJ, Provencal S and Ozonoff S. Social skills interventions for the autism spectrum: essential ingredients and a model curriculum. Child Adolesc Psychiatr Clin N Am. 2003; 12:107-22. | Article | PubMed

11. Rao PA, Beidel DC and Murray MJ. Social skills interventions for children with Asperger's syndrome or high-functioning autism: a review and recommendations. J Autism Dev Disord. 2008; 38:353-61. | Article | PubMed

12. Williams White S, Keonig K and Scahill L. Social skills development in children with autism spectrum disorders: a review of the intervention research. J Autism Dev Disord. 2007; 37:1858-68. | Article | PubMed

13. Reichow $B$ and Volkmar FR. Social skills interventions for individuals with autism: evaluation for evidence-based practices within a best evidence synthesis framework. J Autism Dev Disord. 2010; 40:149-66. | Article | PubMed

14. Bellini S, Peters J, Benner L and Hopf A. A meta-analysis of school based social skillsinterventions for children with autism spectrum disorders. Remedial Spec Educ. 2007; 28:153-162. | Article

15. Forness SR and Kavale KA. Treating social skill deficits in children with learning disabilities: a meta-analysis of the research. Learning
Disability Quarterly. 1996; 19:2-13. | Article

16. Gresham FM, Sugai $G$ and Horner RH. Interpreting outcomes of social skills training for students with high-incidence disabilities. Except Child. 2001; 67:331-344. | Article

17. Mathur SR, Kavale KA, Quinn MM, Forness SR and Rutherford RB $J$ r. Social skills interventions with students with emotional and behavioral problems: a quantitative synthesis of single-subject research. Behavioral Disorders. 1998; 23:193-201. | Article

18. Quinn MM, Kavale KA, Mathur SR, Rutherford RB Jr and Forness SR. A meta-analysis of social skills interventions for students with emotional and behavioral disorders. Journal of Emotional and Behavioral Disorders. 1999; 7:54-64. | Article

19. Reichow B, Steiner AM and Volkmar F. Cochrane review: social skills groups for people aged 6 to 21 with autism spectrum disorders (ASD). Evid Based Child Health. 2013; 8:266-315. | Article | PubMed

20. Wang S, Cui Y and Parrilla R. Examining the effectiveness of peermediated and video-modeling social skills interventions for children with autism spectrum disorders: a meta-analysis in single-case research using HLM. Res Autism Spectr Disord. 2011; 5:562-569. | Article

21. Wang SY, Parrila R and Cui Y. Meta-analysis of social skills interventions of single-case research for individuals with autism spectrum disorders: results from three-level HLM. J Autism Dev Disord. 2013; 43:1701-16. | Article I PubMed

22. Wang $P$ and Spillane A. Evidence-based social sills interventions for children with autism: a meta-analysis. Educ Train Dev Disabil. 2009; 44:318-342. | Pdf

23. Bellini S. Building social relationships 2: a systematic approach to teaching social interaction skills to children and adolescents with autism spectrum disorders and other social difficulties. Shawnee Mission, KS: Autism Asperger Publishing. 2016.

24. Gresham FM. Best practices in social skills training. Bethesda,MD:NationalAssociation of SchoolPsychologists; 2002 . 1029-1040 p.(Thomas A, Grimes J, editors. Best practicesinschoolpsychology. $4^{\text {th }} \mathrm{ed}$ ).

25. Pressley $\mathrm{M}$ and McCormick C. Cognition, teaching and assessment. New York: Harper Collins College Publishers; 1995.

26. Schonert-Reichl KA, Kitil MJ and Hanson-Peterson J. To reach the students, teach the teachers: A national scan of teacher preparation and social and emotional learning. A report prepared for the Collaborative for Academic, Social, and Emotional Learning (CASEL). Vancouver, B.C.: University of British Columbia. 2017.

27. Gresham FM and Lambros KM. Behavioral and functional assessment. Watson TS, Gresham FM, editors. New York: Plenum Press. 1998; 3-22.

28. Bellini S. Building social relationships: a systematic approach to teaching social interaction skills to children and adolescents with autism spectrum disorders and other social difficulties. Shawnee Mission, KS: Autism Asperger Publishing. 2006.

29. Bellini S, Benner L and Peters-Myszak J. A systematic approach to teaching social skills to children with autism spectrum disorders: A guide for practitioners. Beyond Behavior. 2009; 19:26-39. | Article

30. Wong C, Odom SL, Hume KA, Cox AW, Fettig A, Kucharczyk S, Brock ME, Plavnick JB, Fleury VP and Schultz TR. Evidence-Based Practices for Children, Youth, and Young Adults with Autism Spectrum Disorder: A Comprehensive Review. J Autism Dev Disord. 2015; 45:1951-66. | Article I PubMed

31. NationalAutismCenter (US). Nationalstandardsreport, phase 2. 2019. | Website

\section{Citation:}

Bellini $S$ and Heck $O$. The Ingredients of Effective Social Skills Programming for Children and Adolescents on the Autism Spectrum: A Synthesis of Meta-Analytical Research. J Autism. 2019; 6:3. http://dx.doi.org/10.7243/2054-992X-6-3 